\title{
The search for an effective malaria vaccine
}

The report of a recent workshop on vaccines against malaria is sub-titled 'hope in a gathering storm' [1]. The elements of the storm are easy to recognise. Globally, there are around 500 million clinical cases of malaria each year and 2-3 million deaths, most of these young children in Africa south of the Sahara. Plasmodium falciparum malaria is the killer, but there is an additional and massive burden as a result of clinical disease caused by all of the malaria species that infect man. The situation is deteriorating in many areas as a result of the problems presented by increasing resistance to antimalarial drugs. The most hopeful strategy in recent years has been to reduce biting by the mosquito vector by use of insecticideimpregnated bednets and curtains, but even here efficacy is lower where transmission is intense, and there are concerns that use of the nets will lead first to a change in the behaviour of mosquitoes so that they bite out of doors, and secondly to a different pattern of disease, with severe, life-threatening infections being shifted to older children. A vaccine, even one that gave only partial (but reproducible) protection would clearly enhance the effectiveness of the control strategies. Such a vaccine has yet to be developed and this is perhaps not surprising. The parasite has a complex life cycle. The sporozoites injected by an infected mosquito reach the liver parenchyma cells and initiate a cycle of asexual multiplication which increases numbers many thousand-fold. Invasion of erythrocytes gives rise to further cycles of multiplication of the asexual stages that are responsible for the clinical disease and mortality. The blood also contains the sexual stages that are infective to mosquitoes. Each stage of the life cycle is antigenically complex and there is a high degree of stage specificity to the immunity that eventually develops. There is polymorphism among antigens that are recognised immunologically during natural infections, and the parasite has a remarkable capacity for antigenic variation. Model infections, mostly rodent and primate malarias, have been of great value in the development of vaccine strategies but have proved to be of more limited use in predicting the outcome in man after immunisation with similar antigens or formulations.

With one notable exception, the clinical trials in human subjects have been small-scale phase I or phase II trials and these have been directed against the pre-erythrocytic stages, i.e., the infective sporozoites and the liver forms. Early studies showed that radiation-attenuated sporozoites would give good protection against subsequent challenge. As this is an impractical way of vaccinating, extensive studies have been undertaken to attempt to produce a formulation that would reproduce such an immunity. These have been based to a large extent on the circumsporozoite protein, the immunodominant part of which is a long series of tandem repeats of small numbers of amino acids (c. 40 repeats of NANP in $P$. falciparum). Peptide and recombinant forms of the tandem repeats were tested in human volunteers with different adjuvant formulations but with limited success [2]. The primary aim was to induce very high levels of antibodies to the circumsporozoite protein. From subsequent studies it became clear that other effector mechanisms, notably cytotoxic T-cell responses directed against the intrahepatic forms, and induction of high levels of $\gamma$-interferon were probably also required. Later formulations have extended expression of the circumsporozoite protein to include T-helper and cytotoxic T-cell epitope regions that flank the tandem repeats, or provision of T-cell help by use of other antigens, or both. In addition, the antigen has been presented or expressed in different ways, including use of multiple antigen peptides [3], and live viral vectors [4]. Experimentally these have given higher levels of protection, although none is $100 \%$ effective, probably because of polymorphism of T-cell epitopes. Interestingly, a vaccine consisting of the circumsporozoite protein antigen co-expressed in yeast with the hepatitis B surface antigen induced potent cytotoxic $\mathrm{T}$ lymphocyte, $\gamma$-interferon and interleukin-2 responses in animal models. When volunteers were immunised with this construct of $P$. falciparum together with an adjuvant consisting of an oil in water emulsion, monophosphoryl lipid A and Quillaia saponin, six out of seven did not become parasitaemic after challenge by exposure to mosquitoes infected with the homologous strain of the parasite [5]. These are encouraging results, but it remains to be seen whether, in proposed phase II and III trials, the vaccine will be as effective against the multiple heterologous challenge that occurs naturally.

The one vaccine to have been clinically tested on a large scale is a polymerised synthetic peptide, SPf66, the monomer of which consists of short peptides based on the N-terminal sequences of three blood stage antigens of $P$. falciparum plus the NANP repeats of the circumsporozoite protein. Trials in South 
America, notably in Colombia, where malaria transmission is low, gave a reduction in clinical cases of malaria of around $35 \%$ [6]. A broadly similar result was reported from Tanzania, from an area where transmission is intense, when 1-5-year-old children were vaccinated [7]. However, no protection was achieved in The Gambia when infants under 1 year, the age group at highest risk, were vaccinated before the period of seasonal transmission [8], or in Thailand where the vaccinated group consisted of 1-15-year old children [9]. One further trial, in infants in Tanzania, is in progress but it seems likely that, while the concept of a multi-epitope sub-unit vaccine is well worth pursuing, this particular construct is unlikely to be tested more widely.

As the asexual blood stage of infection is responsible for clinical malaria, considerable efforts are being made to select antigens that will induce a protective immune response directed either against the merozoite that invades the erythrocyte or against the greatly modified surface of an infected red blood cell. The evidence to support some of the antigens being vaccine candidates is not strong; in other cases there are promising results from trials in primates and from epidemiological studies. Of the single antigens being investigated, immune responses to the merozoite surface protein MSP-1, or more specifically the Cterminal MSP-1 19 fragment that remains on the merozoite after the antigen is processed by the parasite, correlate with protective immunity [10]. No clinical trials have been conducted so far but these will happen soon with combinations of three other antigens, apical merozoite antigen-1 (AMA-1), merozoite surface protein 2 (MSP-2) and Pf155/RESA.

An immune response that prevents mosquitoes becoming infected, a so-called transmission-blocking immunity, could have a significant public health impact in endemic areas. Experimentally it is possible to induce a very strong antibody-mediated transmission-blocking immunity to antigens in the sexual cycle before fertilisation or to post-fertilisation target antigens. The end result is the same in each case; there is no malarial oocyst development on the mosquito midgut and hence no production of infective sporozoites. Progress towards the production of recombinant proteins that induce such an immunity is promising, but only one small-scale phase I safety and immunogenicity trial has been undertaken so far [11]. A transmission-blocking vaccine could be used alone in certain endemic situations but it is envisaged that it would have particular value when used in combination with other control measures, including other malaria vaccines, and is seen as being able to prevent the spread of drug-resistant or vaccine-resistant mutants.

Still other approaches to vaccine development are expected [12]. The multiple gene or multiple antigen approach will be tested in various ways. A recombi- nant attenuated vaccinia virus called NYVAC7 that expresses seven $P$. falciparum proteins (against preerythrocytic, erythrocytic and transmission stages) has been made and first studies initiated [cited in 13]. Other multi-unit approaches include combinations of polymorphic epitopes that are naturally immunogenic or, by contrast, selection of epitopes that are not targets of immunity during infection but are deemed to be vital for parasite success [14]. Epitopes that induce cytotoxic T-cell responses to infected hepatocytes are being studied and again could form a multicomponent vaccine [15]. DNA vaccines are close to use in human trials; plasmids carrying DNA for circumsporozoite protein and other pre-erythrocytic stage antigens have given good protection in model infections when used singly or in combination [13].

We are clearly some way from producing a usable malaria vaccine and there are many known and potential obstacles to success. However, the vaccine technologies being applied to malaria, the greater understanding of the epidemiology, and the well organised facilities to test the putative vaccines give some cause for optimism. A number of new candidate vaccines will go into clinical trials in the next few years.

GEOFFREY A. T. TARGETT, Department of Medical Parasitology, London School of Hygiene \& Tropical Medicine, Keppel Street, London WC1E 7HT

\section{References}

1. Russell PK, Howson CP (eds). Vaccines against malaria: hope in a gathering storm. Washington, DC, National Academy Press. 1996.

2. Jones TR, Hoffman SL. Malaria vaccine development. Clin Microbiol Rev 1994; 7: 303-310.

3. Sinnis P, Nussenzweig V. Preventing sporozoite invasion of hepatocytes. In: Hoffman SL (ed) Malaria vaccine development: a multi-immune response approach. Washington, DC, ASM Press. 1996: 15-33.

4. Rodrigues $\mathrm{M}, \mathrm{Li} \mathrm{S}$, Murata $\mathrm{K}$ et al. Influenza and vaccinia viruses expressing malaria $\mathrm{CD} 8+\mathrm{T}$ and $\mathrm{B}$ cell epitopes. Comparison of their immunogenicity and capacity to induce protective immunity. $J$ Immunol 1994; 153: 4636-4648.

5. Stoute JA, Slaovi M, Heppner DG et al. A preliminary evaluation of a recombinant circumsporozoite protein vaccine against Plasmodium falciparum. $N$ Engl $J$ Med 1997; 336: 86-91.

6. Amador R, Aponte JJ, Patarroyo ME. Development and fieldtesting of the synthetic SPf66 malaria vaccine. In: Hoffman SL (ed) Malaria vaccine development: a multi-immune response approach. Washington, DC, ASM Press. 1996: 229-248.

7. Alonso PL, Smith T, Armstrong Schellenberg JRM et al. Randomised trial of efficacy of SPf66 vaccine against Plasmodium falciparum malaria in children in southern Tanzania. Lancet 1996; 344: 1175-1181.

8. D'Alessandro U, Leach A, Drakeley $\mathrm{C}$ et al. Efficacy trial of malaria vaccine SPf66 in Gambian infants. Lancet 1995; 346: 462-467.

9. Nosten F, Luxemburger C, Kyle DE et al. Randomised doubleblind placebo-controlled trial of SPf66 malaria vaccine in children in northwestern Thailand. Lancet 1996; 348: 701-707. 
10. Holder AA. Preventing merozoite invasion of erythrocytes. In Hoffman SL (ed) Malaria vaccine development: a multiimmune response approach. Washington, DC, ASM Press. 1996: 77-104.

11. Kaslow DC. Transmission-blocking vaccines. In: Hoffman SL (ed) Malaria vaccine development: a multi-immune response approach. Washington, DC, ASM Press. 1996: 181-227.

12. Targett GAT. Malaria - advances in vaccines. Curr Opin Infect Dis 1995; 8: 322-327.

13. Hoffman SL, Franke ED, Hollingdale MR, Druilhe $P$
Attacking the infected hepatocyte. In: Hoffman SL (ed) Malaria vaccine development: a multi-immune response approach. Washington, DC, ASM Press. 1996: 35-75.

14. Conway D. Natural selection on polymorphic malaria antigens and the search for a vaccine. Parasitology Today 1997; 13: 26-29.

15. Aidoo M, Lalvani A, Allsopp CEM et al. Identification of conserved antigenic components for a cytotoxic $\mathrm{T}$ lymphocyteinducing vaccine against malaria. Lancet 1995; 345: 1003-1007. 